\title{
Treatment of an ostial and a bifurcation lesion with a new directional atherectomy device
}

\author{
L Favero, J B Simpson, B Reimers
}

Heart 2004;90:e46 (http://www.heartjnl.com/cgi/content/full/90/8/e46). doi: 10.1136/hrt.2004.036095

Two cases of directional coronary atherectomy performed with a new 8 French monorail device for selective plaque excision are illustrated. This report underlines the technical characteristics of this new device, which allows the negotiation of complex coronary anatomy and emphasises the potential utility of directional coronary atherectomy in bifurcation and ostial lesions.

W describe the use of the SilverHawk ( $\mathrm{SH}$ ) system (Fox Hollow Technologies Inc, Redwood City, California, USA), a new device for selective plaque excision, in the treatment of an ostial and a bifurcation lesion.

\section{CASE REPORTS Patient 1}

A 63 year old man with recent onset of effort angina had coronary angiography showing a severe stenosis of the ostium of the ramus intermedius (RI) (fig l, left) and a severe stenosis of the mid left circumflex artery. After administration of $7500 \mathrm{U}$ of unfractionated heparin, a 0.014 inch medium support guidewire (Balance Middleweight, Guidant, Temecula, California, USA), was advanced into the RI through an 8 French JL 4 guiding catheter (Cordis, Warren, New Jersey, USA). Without predilatation, the SH catheter was easily advanced into the RI and six consecutive passes and cuts were made at the ostium (fig 1, middle) by rotating the $\mathrm{SH}$ catheter after each pass to obtain a circumferential removal of the plaque. After plaque excision alone, the residual diameter stenosis was $20 \%$ and TIMI (thrombolysis in myocardial infarction) flow was normal. No further treatment was performed (fig 1, right). Subsequently a stent was implanted in the mid left circumflex artery. Procedural time was 45 minutes and fluoroscopy time 15 minutes. Creatine kinase $\mathrm{MB}$ and troponin $\mathrm{T}$ concentrations did not rise above the upper normal limits after the procedure. The patient was discharged two days later and was asymptomatic in the following six months. The six month control coronary angiography showed the persistence of a good angiographic result at the site of previous interventions.

\section{Patient 2}

A 65 year old man admitted to the coronary care unit because of a non-Q wave myocardial infarction had coronary angiography showing a moderate lesion of the mid right coronary artery and a severe stenosis of the distal right coronary artery at the crux cordis bifurcation involving both the origin of the posterior descending artery and the origin of the right posterolateral branch (fig 2, left). After administration of $7500 \mathrm{U}$ of unfractionated heparin, an 8 French JR 4 guiding catheter (Cordis) was inserted and a 0.014 inch floppy guidewire (Balance Middleweight) was positioned in the posterior descending artery. Without predilatation, the SH catheter was advanced to the posterior descending artery and six passes and cuts were made on the crux cordis and the origin of the posterior descending artery. The guidewire was then retrieved from the posterior descending artery and advanced into the posterolateral branch. Six passes and cuts were made to treat the crux cordis and the origin of the posterolateral branch (fig 2, middle). At the end of atherectomy the angiogram showed TIMI 3 flow in the right coronary artery with a $20 \%$ residual diameter stenosis and a type A dissection at the crux (fig 2, right). No further interventions were performed. After the percutaneous coronary intervention the patient was asymptomatic. Creatine kinase MB and troponin T did not increase. The patient was discharged three days after the procedure and remained asymptomatic in the following six months. Six months after the index procedure, a control coronary angiography showed the persistence of angiographic success at the site of atherectomy with no significant restenosis.

\section{DISCUSSION}

Despite the questionable role of atherectomy in reducing the incidence of restenosis, this technique may still have an important impact on the treatment of ostial and bifurcation lesions. ${ }^{1-4}$ In these kinds of lesions, the removal of plaque potentially enhances the immediate procedural success by reducing plaque shift, which is responsible for side branch closure or vessel compromise. ${ }^{5}$ In patient 1 we regarded plaque excision as the preferred treatment because both balloon angioplasty and stenting presented a very high risk of jeopardising the ostium of the left anterior descending artery, the mid left circumflex, or both. The bifurcation lesion of the crux obviously could have been treated with elective stent implantation. However, bifurcation stenting, even with the use of drug eluting stents, is a complex procedure that is hampered by a considerable incidence of restenosis (Colombo, American Heart Association, 75th Scientific Sessions, 2002). Therefore, excising plaque with conditional stenting may be a strategy for bifurcation lesions to improve immediate and follow up results. The clinical importance of the $\mathrm{SH}$ device for the treatment of bifurcations is also reflected by the fact that in a recent registry of this device, $36 \%$ of treated lesions were bifurcations. ${ }^{6}$ Previous directional atherectomy devices required an occlusive balloon dilatation that pushed the cutter into the plaque. The SH catheter is not based on occlusive balloon inflation but on a longitudinal cutting process. This is achieved by advancing the deflected catheter tip with the activated cutter through the lesion allowing the spinning blade to shave material from the plaque. This concept reduces the "Dotter" and balloon angioplasty effects observed with previous atherectomy

Abbreviations: RI, ramus intermedius; SH, SilverHawk; TIMI, thrombolysis in myocardial infarction 

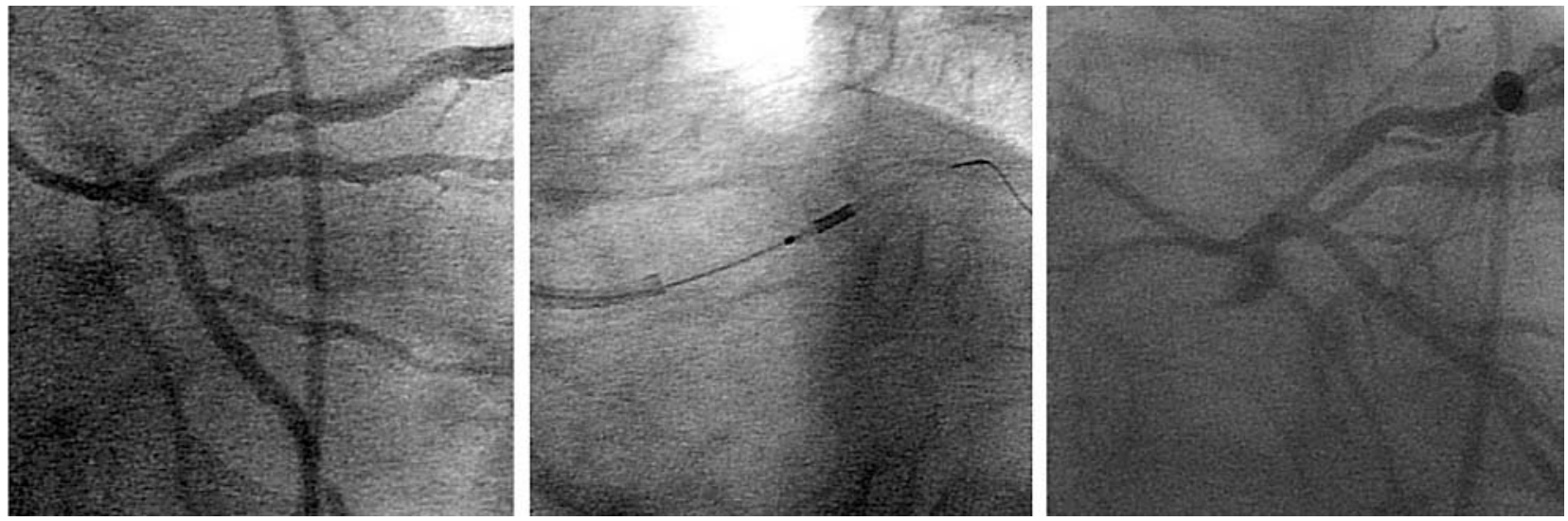

Figure 1 Severe stenosis of the ostium of the ramus intermedius (left). Plaque excision with the SilverHawk catheter at the ostium of ramus (middle). Final angiographic result after plaque excision (right).
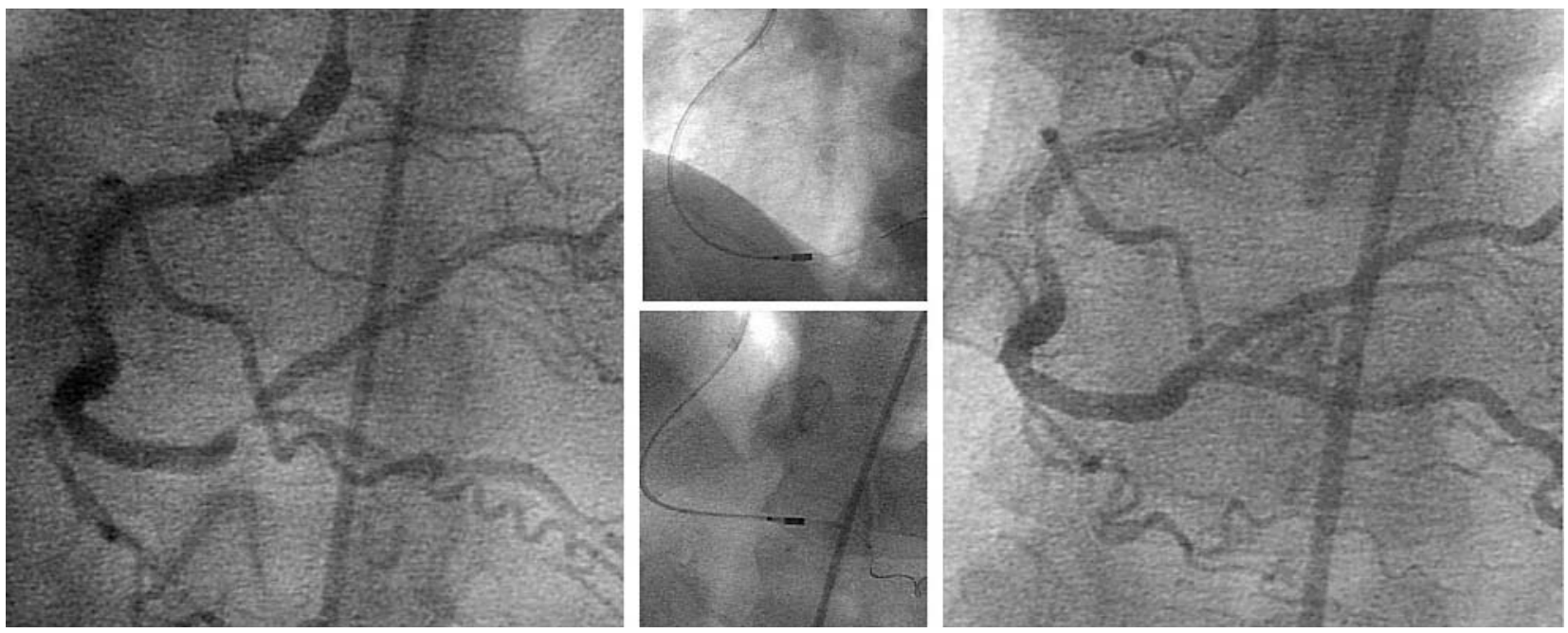

Figure 2 Severe stenosis of the distal right coronary artery at the crux cordis (left). Plaque excision at the crux cordis (middle) towards the posterior descending artery (bottom) and towards the posterolateral branch (top). Final angiographic result after plaque excision (right).

devices and reduces the possibility of plaque shift. In the case of treatment of the ostial lesion atherectomy effectively avoided any compromise of the other branches (fig l, right). In the bifurcation lesion the debulking device was used to treat both branches with no plaque shift observed (fig 2, right). Further features of the $\mathrm{SH}$ system are the more flexible shaft, the shorter stiff cutting section, and the lower profile-technical characteristics that allow negotiation of complex anatomy. With this regard, in patient 2 the $\mathrm{SH}$ catheter was easily advanced through a moderately tortuous right coronary artery to its distal portion by using a conventional medium support percutaneous transluminal coronary angioplasty guidewire. The new concept of selective excision also allows for treatment of smaller vessels. In both of the presented cases an effective excision was achieved in vessels of diameter $\leqslant 3.0 \mathrm{~mm}$. Interestingly in the presented cases plaque debulking was not followed by stent implantation. This, however, was not because atherectomy is seen as an alternative to stent implantation but because of the good angiographic result obtained with only plaque excision and because of the known limitations of stents in ostial and bifurcation lesions situated in small vessels.

In conclusion, the newly designed $\mathrm{SH}$ system may offer significant utility in the treatment of complex coronary lesions potentially alleviating the limitations of conventional directional atherectomy devices.

\section{Authors' affiliations}

L Favero, B Reimers, Cardiology Department, Mirano Hospital, Mirano, Italy

J B Simpson, Stanford University, Stanford, California, USA

Dr Simpson has the following relationships with the product and manufacturer investigated in this paper: he has a financial interest in the Fox Hollow Technologies Inc and is involved in new product development for Fox Hollow Technologies Inc.

Correspondence to: Dr Bernhard Reimers, Cardiology Department, Mirano Hospital, Via Mariutto, 13, 30035 Mirano, Italy; ulss13mir.card@tin.it

\section{Accepted 17 March 2004}

\section{REFERENCES}

1 Moussa I, Moses J, Di Mario C, et al. Stenting after optimal lesion debulking (SOLD) registry: angiographic and clinical outcome. Circulation 1998;98:1604-9.

2 Colombo A, on behalf of the AMIGO investigators. Atherectomy before MULTI-LINK improves luminal gain and clinical outcomes (AMIGO): a comparison of coronary stenting with or without adjunctive directional atherectomy (late-breaking clinical trials). J Am Coll Cardiol 2002;40:1-18. 
3 Karvouni E, Di Mario C, Nishida T, et al. Directional atherectomy prior to stenting in bifurcation lesions: a matched comparison study with stenting alone. Catheter Cardiovasc Interv 2001;53:12-20.

4 Daverman HL, Higgins PJ, Sparano AM, et al. Mechanical debulking versus balloon angioplasty for the treatment of true bifurcation lesions. J Am Coll Cardiol 1998:32:1845-52.
5 Takagi T, Di Mario C, Stankovic G, et al. Effective claque removal with a new 8 French-compatible atherectomy catheter. Catheter Cardiovasc Interv 2002;56:452-9.

6 Orlic D, Reimers B, Stankovic $G$, et al. Initial experience with a new 8 French compatible directional atherectomy catheter: immediate and mid-term results. Catheter Cardiovasc Interv 2003;60:159-66. 